Sādhanā Vol. 38, Part 4, August 2013, pp. 707-722. (C) Indian Academy of Sciences

\title{
The problem of clear air turbulence: Changing perspectives in the understanding of the phenomenon
}

\author{
T N VENKATESH ${ }^{1, *}$ and JOSEPH MATHEW ${ }^{2}$ \\ ${ }^{1}$ Flosolver Unit, CSIR-National Aerospace Laboratories, PB 1779, \\ Bangalore 560 017, India \\ ${ }^{2}$ Department of Aerospace Engineering, Indian Institute of Science, \\ Bangalore 560 012, India \\ e-mail: tnv@flosolver.nal.res.in; joseph@aero.iisc.ernet.in
}

MS received 11 October 2011; revised 24 June 2013; accepted 1 July 2013

\begin{abstract}
Due to rapid improvements in on-board instrumentation and atmospheric observation systems, in most cases, aircraft are able to steer clear of regions of adverse weather. However, they still encounter unexpected bumpy flight conditions in regions away from storms and clouds. This is the phenomenon of clear air turbulence (CAT), which has been a challenge to our understanding as well as efforts at prediction. While most of such cases result in mild discomfort, a few cases can be violent leading to serious injuries to passengers and damage to the aircraft. The underlying physical mechanisms have been sought to be explained in terms of fluid dynamic instabilities and waves in the atmosphere. The main mechanisms which have been proposed are: (i) Kelvin-Helmholtz instability of shear layers, (ii) waves generated from flow over mountains, (iii) inertia-gravity waves from clouds and other sources, (iv) spontaneous imbalance theory and (v) horizontal vortex tubes. This has also undergone a change over the years. We present an overview of the mechanisms proposed and their implications for prediction.
\end{abstract}

Keywords. Clear air turbulence; aviation weather hazards; fluid dynamic instabilities; atmospheric waves.

\section{Introduction}

Atmospheric effects on aviation are well-known and, with the increase of air-traffic, the impacts of weather are being seen more and more. Besides passenger comfort and maintenance of schedules, safe air travel depends significantly on weather phenomena. A US NTSB survey showed

*For correspondence 
that weather is a predominant cause of aviation accidents, accounting for approximately $57.1 \%$ fatal and 27.0\% non-fatal incidents (Mahapatra 1999). An impetus for renewed interest in aviation weather hazards is the loss of Air France flight AF447 of 1 June 2009 over the Atlantic ocean in which all 228 people onboard the Airbus A330, died. According to NCAR, a possible cause of the crash is that the plane encountered severe weather conditions as it flew around intense storms as suggested by an analysis of satellite data. There are many kinds of aviation-related weather phenomena which are of great interest to fluid dynamicists from a research point of view such as, clear air turbulence, wind shear and wake-vortex effects. Here, we examine current understanding of clear air turbulence (CAT) to suggest new directions for research and development, especially for simulations and predictions of hazardous weather phenomena of significance to general aviation.

Clear air turbulence is a phenomenon which still eludes our understanding and methods of detection even after decades of study (Pao \& Goldburg 1969; Knox et al 2008). It is also relevant to aircraft travelling into and out of India. On 25 April 2010, an Emirates flight from Dubai to Kochi, encountered severe turbulence resulting in injuries to several passengers (The Times of India, 26 April 2010). On 26 May 2010, the Air India flight IX-212 dropped a few thousand feet on encountering an 'air-pocket' (PTI News, 31 May 2010). These incidents underscore the importance of understanding such phenomena. While there are many papers on various aspects of CAT, in recent times, there has not been any comprehensive review of CAT. In this survey, first we describe observations about CAT events and attempts at detection. The discussion that follows on mechanisms that have been proposed for the formation and sustenance of CAT is the major emphasis of this paper, since it is the starting point for future research and improved understanding. Finally, we consider forecasting methods.

\subsection{Definition of clear air turbulence}

Clear air turbulence (CAT) can be defined as 'All turbulence in the free atmosphere of interest in aerospace operations that is not in, or adjacent to visible convective activity. This includes

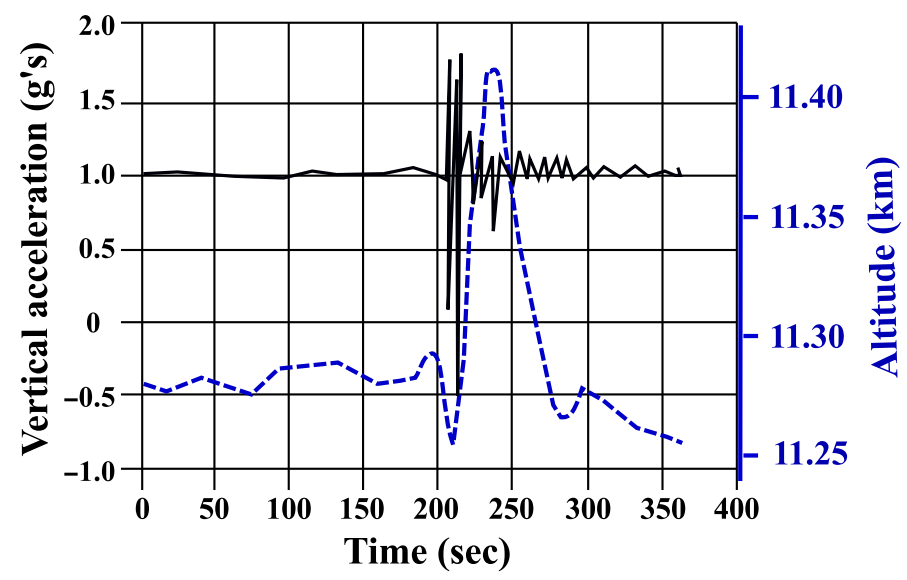

Figure 1. Time history of vertical accelerations (solid) and altitude (dashed) along the flight path of a commercial jet aircraft near Hannibal MO, from 0121 to 0127 UTC on 4 April 1981. Adapted from figure 4 of Ellrod et al (2003). 
turbulence found in cirrus clouds not in or adjacent to visible convective activity' (Pao \& Goldburg 1969). The above could also be expressed as 'All bumpy flight conditions away from convective clouds'. CAT is also defined as aircraft turbulence that occurs at altitudes of $5.6 \mathrm{~km}$ $(500 \mathrm{hPa})$ or higher. This definition excludes many of the low-level disturbances, which can be attributed to the boundary layer or topographic effects, such as mountain lee-waves. An example of the vertical acceleration encountered by an aircraft due to CAT is shown in figure 1. One can see that the aircraft was subjected to significant acceleration and altitude change of a few hundred metres during a short time (around 100 seconds).

Bumpy flight conditions could be due to high vertical velocities and gusts associated with either clouds/storms or those appearing in clear air. With the advances in radar technology, regions of convective activity are detected easily and aircraft can avoid such areas. The more dangerous of the two is CAT since it cannot be detected by current weather radars. Currently research is being carried out to detect such regions using satellite observations (Donovan et al 2008). Based on the magnitude of the gusts, regimes are classified as light $(1.5-3.0 \mathrm{~m} / \mathrm{s})$, moderate $(3.0-6.1 \mathrm{~m} / \mathrm{s})$ or severe $(>6.1 \mathrm{~m} / \mathrm{s})$ (Endlich 1964). The size of the turbulent zone can also be significant (161 km wide, $1.5 \mathrm{~km}$ deep) and may persist for around $21 / 2 \mathrm{~h}$.

\section{Observations}

CAT is generally associated with mesoscale (length-scale of around 10-50 km and time-scale of a few hours) phenomena, such as jet-streams, troughs, ridges, fronts. It could also be embedded in synoptic flow (length scale of around $1000 \mathrm{~km}$ and time-scale of 1-2 days) and, so, persists for many hours. Observational studies using research aircraft have indicated that the presence of high clouds, vertical wind shear, wind speed, presence of jet-stream (a band of high speed winds, blowing from east to west, at altitudes of $5 \mathrm{~km}$ or more) and Richardson number (ratio of buoyancy to shear) have a high correlation with CAT (Aiken and Lean 1973). Other parameters like time of day, flight direction, height and temperature of tropopause were found to have no significant relation with CAT (Colson 1963). Atlas et al (1970) observed the formation, growth and breakup of a Kelvin-Helmholtz wave, in a manner similar to that observed in laboratory shear flows, near a region associated with turbulence. CAT is more often found near the jetstream. It is more frequently observed near mountains and continents than over flat land and oceans. In the absence of a satellite based method of detection, these reports could be biased by the aircraft flight paths and the limited regions of study.

Necessary and sufficient conditions for CAT have not been isolated but some of the factors associated with it are:

(i) $\mathrm{Ri}<1$; (Ri, the Richardson number, is the ratio of buoyancy forces to shear forces. A value less than one indicates that shear instabilities dominate).

(ii) Discontinuities of lapse rate; (The lapse rate is the rate at which temperature decreases with height in the atmosphere.)

(iii) Large cyclonic horizontal shear.

(iv) Large vertical velocities $(\sim 1 \mathrm{~m} / \mathrm{s})$.

In flights using research aircraft, CAT has been observed to have a 'patchy nature' (Reiter in Pao \& Goldburg (1969)). The relevant scales are: altitude above 5 km; extent of 20-200 m, and durations of the order of seconds. 


\subsection{Detection}

Ground-based radars with sufficient power, such as MST (Mesosphere-StratosphereTroposphere) radar which uses the Doppler effect to measures wind velocities, can detect gusts and can in principle be used to infer regions of CAT. However, these have a limited range, and can be used for research studies but are not suitable for practical detection/avoidance. Weather radars on aircraft can detect water droplets or ice-crystals and thus, rain and convective activity. Particle sizes of fog/cloud, etc. are too small to be detected by this type of radar. Turbulence in clear air has no large particles associated with it and cannot be detected by airborne radar.

A lidar (laser-based light detection and ranging) detects density differences and can be used for CAT avoidance. So far, such instruments have been used on ground based platforms and research aircraft only. Lidars have not been used on commercial aircraft due to power and cost considerations. Kaufmann (2002) has examined the economics of fitting such instruments onboard and expected benefits. From the figures quoted, it appears unlikely that airlines will be using lidars in the near future.

Pilot reports (PIREPs) remain the most reliable locators of CAT. When an aircraft encounters a region of turbulence, the pilot informs the ground control about the location and extent. This information is passed on to other aircraft in the region. Pilots are also expected to record such incidents after landing. Databases based on PIREPs have been used by many researchers to validate their models (Knox et al 2008). However, this practice is airline/country-dependent and not universally followed. CAT reports are not available at all parts of the world. A limitation of PIREP-based CAT database is that there are no data on regions away from major air-routes.

A satellite based method of CAT detection would provide a global coverage of the phenomenon. However, the spatial resolution of geo-stationary satellites is not sufficient to detect CAT, while with low-earth satellites the temporal sampling rate is low. Some new, indirect techniques or combination of data from many low-earth satellites could solve this problem in the future.

\section{Theories for formation}

The earth's boundary layer is predominantly turbulent, and has been studied extensively. The surface layer is explained by the Monin-Obukhov theory and heat-flux scaling in the low wind regime. In the free atmosphere, well above the the boundary layer, the level of turbulence is expected to be low as there is a rapid decay of turbulence with height. Also, in regions away from clouds stratification would be stable, and this would further reduce the turbulence levels. This suggests that shear instabilities or wave propagation could be the likely sources of CAT. The several candidate mechanisms that have been studied in the past are discussed here. It is certainly possible that the different mechanisms are appropriate to different topography and atmospheric conditions even though the result is labelled a CAT event.

\subsection{Kelvin-Helmholtz instability}

Most theoretical analyses, done in the 60s and 70s, considered CAT to arise from a KelvinHelmholtz type of instability from shear layers in the atmosphere. A conceptual view of the process is shown in figure 2. Atlas et al (1970) have observed the formation, growth, and breakup of a Kelvin-Helmholtz wave, in a manner similar to that observed in laboratory shear flows, near a region associated with turbulence. 
a

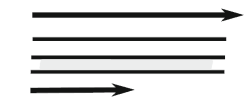

c

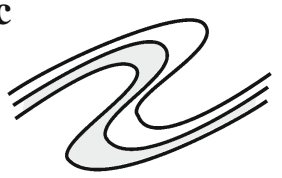

e

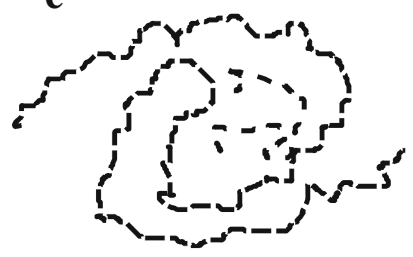

b
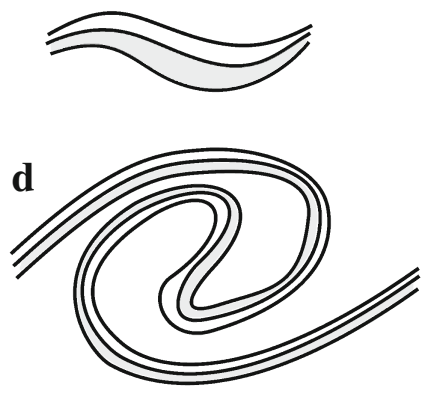

$\mathbf{f}$

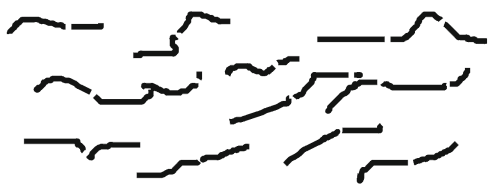

Figure 2. Schematic diagram of the instability of a shear layer and breakdown into turbulence. Adapted from figure 2 of Ellrod \& Knapp (1992).

Kelvin-Helmholtz instability is the linear instability at the interface of two streams. For two streams of fluid of different densities $\rho_{1}, \rho_{2}$ and speeds $U_{1}, U_{2}$ separated by a horizontal interface, under the assumptions of no viscosity, the wave speed of small perturbations,

$$
c=\frac{\rho_{1} U_{1}+\rho_{2} U_{2}}{\left(\rho_{1}+\rho_{2}\right)} \pm \sqrt{-\frac{\rho_{1} \rho_{2}\left(U_{1}-U_{2}\right)^{2}}{\left(\rho_{1}+\rho_{2}\right)^{2}}-\frac{\rho_{2}-\rho_{1}}{\left(\rho_{1}+\rho_{2}\right)} \frac{g}{k}} .
$$

Here, $g$ is the acceleration due to gravity and $k$ the wavenumber. If there is no velocity difference, the configuration is unstable if $\rho_{2}>\rho_{1}$, i.e., the heavier fluid is on top (Drazin 2002). If there is a velocity difference, the flow is unstable for high wavenumbers

$$
k>\frac{\rho_{1}^{2}-\rho_{2}^{2}}{\rho_{1} \rho_{2}\left(U_{1}-U_{2}\right)^{2}} g .
$$

Thorpe $(1968,1971,1973)$ conducted laboratory studies on the mixing of stably stratified fluids and the Kelvin-Helmholtz instability. They found that internal waves affect the density gradient, creating unstable regions eventually leading to turbulence. They also observed turbulence generated in the interior of a stratified layer (not at the interface). A comparison of the parameters with scales in the atmosphere and oceans showed that the non-dimensional collapse time of $\mathrm{K}-\mathrm{H}$ billows is similar for flows in the laboratory and those in the atmosphere or oceans.

Other effects such as (i) surface tension, (ii) viscosity, (iii) rotation have been studied by Chandrasekhar (1961). Viscosity and rotation have a stabilizing effect and impose a cutoff wavenumber but higher wavenumbers are still unstable. Surface tension will suppress the instability if

$$
\left(U_{1}-U_{2}\right)^{2}<\frac{2\left(\rho_{1}+\rho_{2}\right)}{\rho_{1} \rho_{2}} \sqrt{\operatorname{Tg}\left(\rho_{1}-\rho_{2}\right)},
$$


where $T$ is the surface tension. Surface tension is important for high curvature, or high wavenumbers, and is significant for the air-sea interface, but not for fronts in the atmosphere (Chandrasekhar 1961).

If there is continuous stratification and the velocity varies as a function of height, the HowardMiles criterion for instability is

$$
R i<\frac{1}{4},
$$

based on the Richardson number $\left(R i=N^{2} /\left(U^{\prime}\right)^{2}\right.$, where $N^{2}=-(g / \bar{\rho}) d \bar{\rho} / d z$ is the BruntVaisala frequency and $U^{\prime}=d U / d z$ ) (Howard 1961; Miles 1961). This criterion was initially used as predictor of CAT. However, large regions of with low values of $R i$ can exist without CAT being triggered and it is now regarded as only a necessary condition for CAT.

\subsection{Mountain flows}

Mountains act as obstacles to the flow and are major sources of generation of mesoscale waves. Complex terrain can lead to formation of rollers, cloud bands and also generate inertia gravity waves which propagate to large distances.

Pao \& Goldburg (1969) quotes Reiter's statement: 'The turbulence energy within the isotropic sub-range may be fed by the kinetic energy of the flow over rough terrain in a near-neutral stratification. This could be the case for low level turbulence. The 'spectrum' of the terrain configuration may have an influence on eddy sizes beyond the inertial sub-range. ... Such flow processes have been poorly explored as yet.'

Belcher \& Hunt (1998), in their review of flow over mountains, use the advective and Lagrangian timescales to divide the flow into an inner and outer region (figure 3). Many aspects of the interactions between the flow, generation of turbulent stresses in the atmosphere and resulting drag on mountains are described. Regarding the effect of hills and waves on large-scale

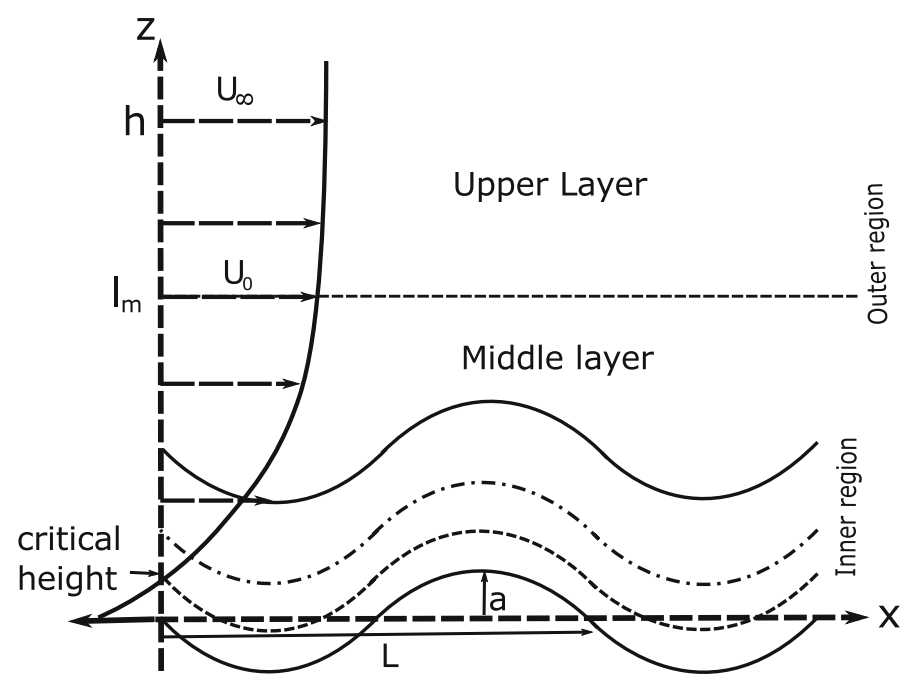

Figure 3. Regions of interest in flow over a range of mountains such as the upper layer, middle layer and inner region. The lower geometry is idealized as a sine wave. Adapted from figure 3 of Belcher \& Hunt (1998). 
eddies, they show that the stream-wise vorticity is increased while vertical vorticity is decreased. When the flow is perpendicular to a mountain range, they show that a secondary flow can result (from a span-wise inviscid instability), with vortices having a vertical scale comparable with the boundary layer depth.

Calculations of flow over a one kilometer high, two-dimensional ridge show that propagating waves can reach the tropopause (Lin 2007). For three-dimensional mountains, the upward propagation is less, but there is a spreading of the disturbance in the horizontal direction. For larger mountains, Coriolis effects are important because they can sustain the generated waves for a longer time/distance (conservation of potential vorticity).

Mountain generated turbulence is definitely the cause of low level disturbances. Whether it causes CAT at cruise altitudes is still not known. A possible mechanism is by the triggering of gravity waves which can propagate over large distances and to higher altitudes.

\subsection{Gravity waves}

While initially the Kelvin-Helmholtz instability was considered to be the primary mechanism for CAT, later, the role of internal inertia-gravity waves (Nappo 2002) gained more importance (Ellrod et al 2003). Inertia-gravity (IG) waves can be triggered by shear zones, flows over mountains or around regions of intense convection (Simpson 1997). In addition, these waves can reflect from the tropopause or ground and cause CAT at locations away from the source of the disturbance. Lindzen (1974) noted that, in the classical K-H problem, shear and stable stratification are confined to a thin layer, and that the unstable perturbations decay away from the shear zone. In a fluid with stable stratification everywhere, it was shown that for low wavenumbers $\left(k^{2}<N^{2} / U^{2}\right)$, inertia-gravity waves, which propagate far from the shear zone, exist. It is their view that these waves 'can supplement and perhaps supplant Kelvin-Helmholtz instability in explaining clear air turbulence'. When $R i_{B}$ is greater than $1 / 4$, instead of instability, wave solutions exist. The dispersion relation is

$$
\omega=\bar{U} k \pm \sqrt{\frac{k^{2} N^{2}}{\left(k^{2}+m^{2}\right)}}
$$

If there is no mean wind, IG waves can propagate at any angle with respect to the horizontal. The highest frequency of oscillation is limited to $N$. An example of vertical velocities encountered by and aircraft, due to IG waves is shown in figure 4.

Since the propagation velocity depends on the mean windspeed, its variations can result in (i) ducting or (ii) reflection of IG waves. Ducting occurs when there is a strong jet in the atmosphere. In the centre of the jet, wave velocity is high and nearly aligned with the jet axis, while at the edges, the velocities are low and towards the jet. This results in IG waves being 'carried along' with the jet over long distances with little attenuation. IG waves can reflect off the ground or from critical layers in the atmosphere. Critical levels are those where $U$ becomes equal to $c$ (since the sign of $U-c$ determines the nature of the solution). Due to ducting and reflection IG waves can transport energy over long distances $(1000 \mathrm{~km})$.

Many authors have held that there is a strong connection between inertia-gravity waves and CAT: One possibility is that these waves 'degenerate' into turbulence. The currently held view is that inertia-gravity waves reduce local $R i_{B}$ and promote growth of instability by Kelvin-Helmholtz mechanism. Observations of regions of mountain induced gravity waves and regions of turbulence, possibly associated with these waves, are shown in figure 5 (from Nappo (2002)). 


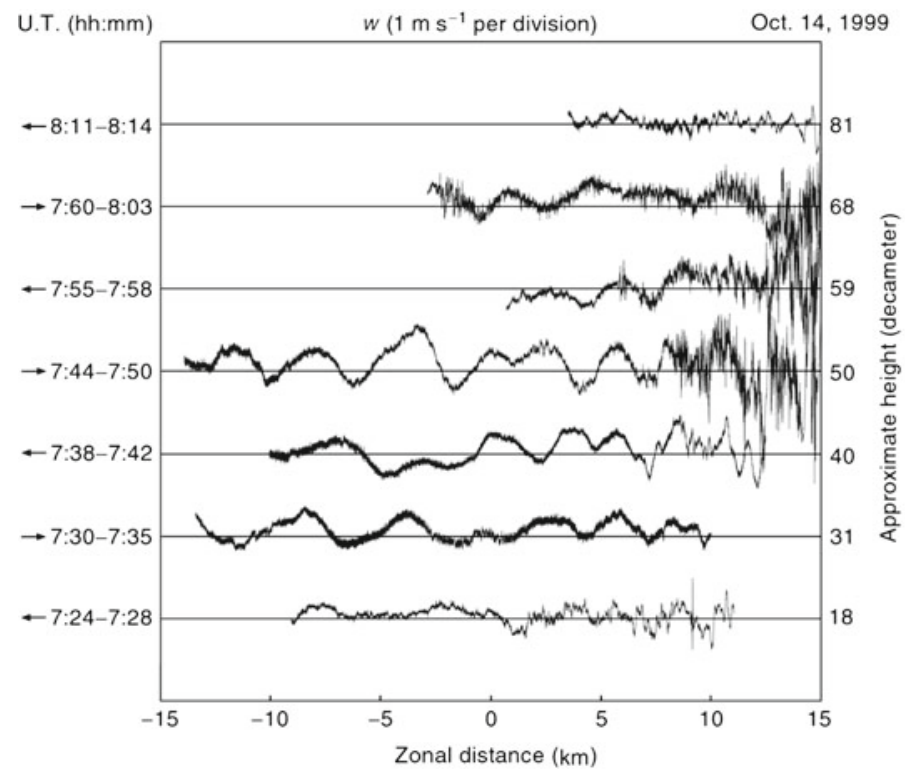

Figure 4. Traces of vertical velocity recorded by a research aircraft, the King-Air B200, during CASES99 field campaign, on 14 October 1999. The flights were made at various heights at a fixed latitude. The flight directions (towards east or towards west) are indicated by the arrows on the left of the figure (Figure 1.3 of Nappo (2002), Academic Press).

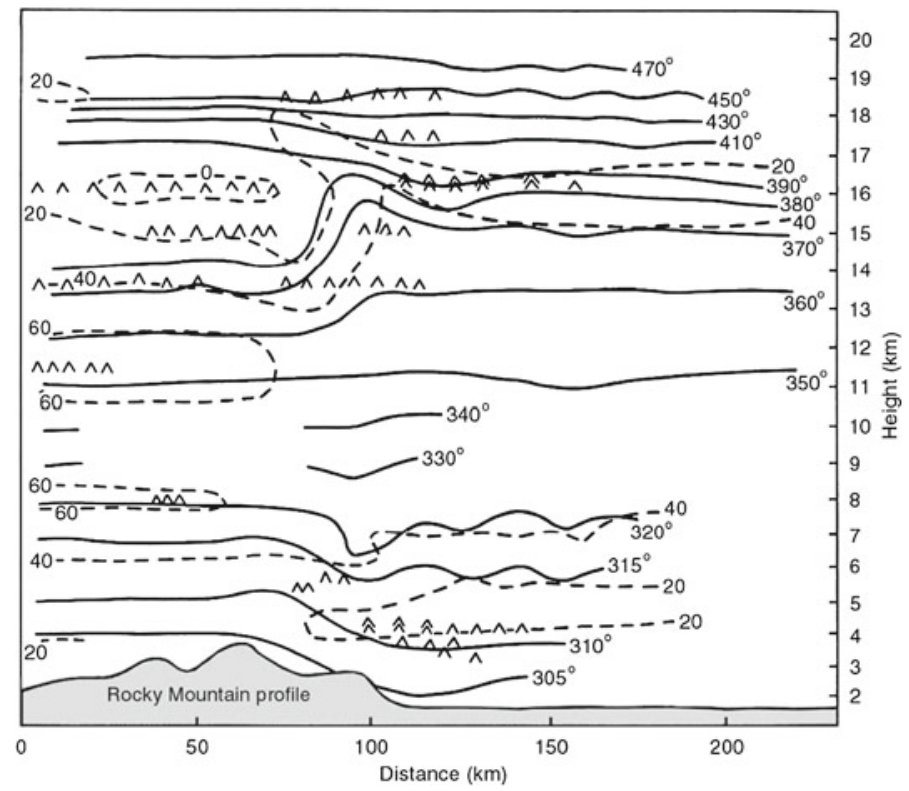

Figure 5. A view of mountain induced gravity waves and regions of turbulence on February 17, 1970, over Colorado. Solid lines represent potential temperature, dashed lines U component of velocity, represents light turbulence and $\hat{\hat{}}$ represents strong turbulence (Figure 3.1 of Nappo (2002), Academic Press). 


\subsection{Lighthill-Ford theory}

Knox (1997) noted that operational forecasts of CAT using conventional diagnostics have poor correlation (0.1-0.35). He analysed the theoretical basis of the various forecasting techniques and advanced simple arguments to show that most of them fail in regions of strong anti-cyclonic flow. He suggested that inertial instability and spontaneous gravity wave generation could be possible mechanisms.

Ford and co-workers (Ford 1994; Ford et al 2000) derived an equation for the generation of inertia-gravity waves from regions of vorticity in a manner similar to Lighthill's derivation for Lighthill's acoustic waves. Starting from the shallow water equations for a layer of thickness $h$, an equation for $\partial h / \partial t$ can be derived as follows.

$$
\left(\frac{\partial^{2}}{\partial t^{2}}+f^{2}-c_{0}^{2} \frac{\partial^{2}}{\partial x_{i} \partial x_{i}}\right)\left(\frac{\partial h}{\partial t}\right)=\frac{\partial^{2}}{\partial x_{i} \partial x_{j}}\left(T_{i j}\right),
$$

where

$$
T_{i j}=\frac{\partial}{\partial t}\left(h u_{i} u_{j}\right)+\frac{g}{2} \frac{\partial}{\partial t}\left(h^{2}-h_{0}^{2}\right) \delta_{i j}+\frac{f}{2}\left[\epsilon_{i k} h u_{k} u_{j}+\epsilon_{j k} h u_{k} u_{i}\right],
$$

where $f=2 \Omega \sin \phi$ is the Coriolis parameter, $\phi$ the latitude, $\Omega$ earth's angular velocity, $h_{0}$ a reference height and $c_{0}=\sqrt{g h_{0}}$.

Knox et al (2008) argue that the Lighthill-Ford theory of spontaneous imbalance, can explain CAT. According to this theory, regions of flow with significant vorticity can emit waves (due to internal small scale fluctuations) and mechanisms such as geostrophic adjustment or topographically generated gravity waves are not necessary. In a similar vein, Medvedev \& Gavrilov (1995), Gavrilov (1997) used asymptotic analysis and method of multiple timescales to derive the source of forcing of IGW.

Plougonven et al (2009) have pointed out that both Lighthill and Ford used the scale separation between the source of the waves (non-zero only in a small region) and the region of propagation of the waves, to make the scaling arguments and that use of the vorticity term as a local indicator of CAT, by Knox et al (2008) does not follow from the theory.

\subsection{Horizontal vortex tubes}

In addition to waves or instabilities, another approach which has been adopted recently, is to look at coherent-structures in the flow associated with CAT (figure 6).

Clark et al (2000) simulated conditions similar to those encountered by a DC- 8 aircraft over Colorado, during December 1992, using a non-hydrostatic model with a resolution from $26 \mathrm{~km}$ to $200 \mathrm{~m}$ (5 levels of nesting). They found that flow-reversal associated with wave breaking occurred two to three $\mathrm{km}$ above the incident. They infer that this region of intense turbulence was due to the breaking of vertically propagating gravity waves. In addition, their simulations suggest that a Horizontal Vortex Tube (HVT) was formed which led to turbulent conditions (as violent as those due to a thunderstorm). Clark \& Radke (2001) further suggest that, HVTs can be triggered by 3-D shear layers.

Oblique encounter of an aircraft with an HVT of similar dimensions, can lead to inversion of the aircraft (if encountered at right angles, only an up-down motion results). The scenario, described by Clark \& Radke (2001) is illustrated in figure 7. First one wing encounters a downward velocity leading to loss of lift. The pilot compensates by increasing the aileron deflection on that wing. By this time, the wing encounters an upward wind while the other wing encounters a downward wind, leading to roll in the opposite direction, which adds to the aileron deflection. 


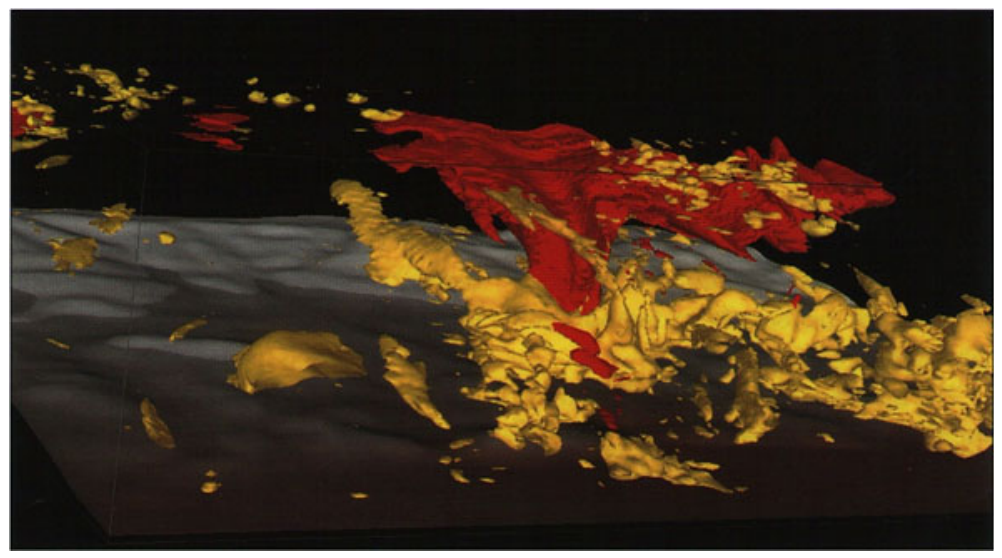

Figure 6. Simulated flow structures near a CAT event (From Clark \& Radke 2001).
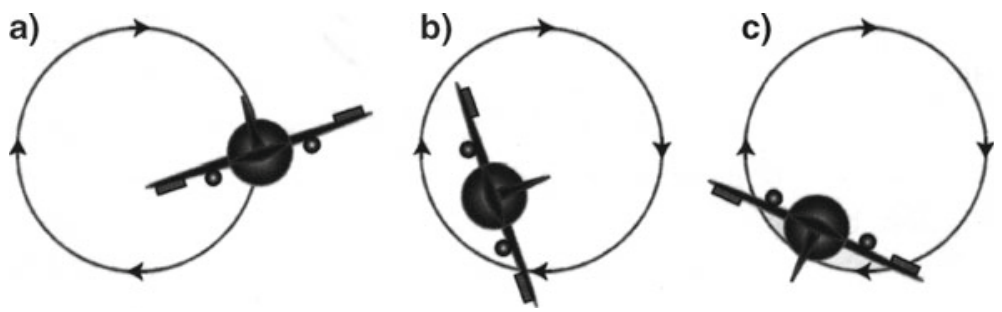

Figure 7. Oblique encounter of an aircraft with a HVT (from Clark \& Radke 2001).

The mechanism proposed for the formation of HVTs is as follows. Jets and fronts in the atmosphere are regions where the shear is high. Kelvin-Helmholtz is one of the instabilities possible in a shear layer. If three-dimensional perturbations are considered over the sheet, instability in the span-wise direction (growth of spanwise perturbations), can also be present leading to the formation of braids - this is usually ignored because the growth rates are smaller. In addition, if the density gradient is sharper than the shear layer, the Holmboe instability is triggered (Smyth \& Winters 2003).

\section{Slow decay of turbulence}

Another aspect of the problem is the manner in which the turbulent fluctuations and gravity waves decay. Once a patch of turbulence is formed, due to any of the mechanisms described in the previous sections, the rate of decay would determine how long it would last.

Dickey \& Mellor (1980) studied the decaying of turbulence in neutral and stratified fluids, experimentally at a mesh Reynolds number of 48260. They observed that for the neutral case decay is a per a power law $t^{-1}$. For the stratified experiment, upto non-dimensional time of 275 , the decay rate was identical to that of the neutral case and subsequently the decay rate sharply decreased. After this time the field of turbulence was replaced by internal gravity waves. 
Weinstock (1984), made a theoretical calculation of the decay of turbulence energy in the presence of coherent internal gravity waves of various intensities. They show that the turbulence decay is a universal function of the wave shear and wave frequency provided that the energy is expressed in terms of the buoyancy wavenumber $k$, and time is expressed in terms of the Brunt-Vaisala frequency. Similar to the experimental result of Dickey \& Mellor (1980), the turbulence decay is found to undergo a sudden transition from rapid decay to a much slower oscillatory decay when the gradient Richardson number, is less than about 0.4 . They suggest that this may explain the continued presence of turbulence in dynamically stable regions of oceans or atmosphere. With rotation also the rate of decay is reduced (Moisy et al 2011).

Decay of vortices in stratified fluids has been studied in the context of aircraft wakes. These studies have been reviewed by Spalart (1998). Presence of strong vortical structures is one of the mechanisms proposed for severe CAT. Therefore some of studies on the decay of vortices in stratified fluids are relevant to the problem of decay of CAT. The overall sequence of events is thought to be as follows: A pair of counter-rotating vortices, shed from an aircraft wing-tips, descend due to mutual induction. Initially they are subject to instabilities: short-wave elliptical and longwave Crow instability, which can lead to breakdown (high dissipation). Later they diffuse with rapid mixing with the ambient atmosphere. There are conflicting views about the time duration of the different phases, the temporal dependence, etc. The modes of decay could be either rapid or slow. Spalart (1996) describes the exponential decay of laminar wakes in a stratified fluid.

\section{Forecasting methods}

There have been a few methods proposed for forecasting clear air turbulence based on largescale observations. Early methods considered the Richardson number to be the primary predictor (Kronebach 1964). Such methods identify large regions of possible occurrence of CAT. The main source of verification of such methods has been using pilot reports (PIREPs) of CAT.

Colson \& Panofsky (1965) used dimensional arguments in a stable atmosphere to estimate clear-air turbulence intensities as follows

$$
C P=\lambda^{2} S_{V}^{2}\left(1-\frac{R i}{R i_{\text {crit }}}\right),
$$

where $\lambda$ is a vertical length scale, $S_{V}$ the vertical shear and $R i_{\text {crit }}$ an empirical constant.

Mancuso \& Endlich (1966) used turbulence data collected during a field campaign during 1962 to find correlations between turbulence frequency and meteorological parameters. They found that the product of vertical vector wind shear and deformation correlated well with regions of CAT.

Ellrod \& Knapp (1992) used a combination of vertical wind shear (VWS), deformation (DEF) and convergence $(\mathrm{CVG})$, based on large-scale observed values of wind to define a turbulence index as follows.

$$
T I 2=V W S \times[D E F+C V G],
$$

where

$$
\begin{gathered}
V W S=\frac{\partial V}{\partial z} \\
C V G=-\left(\frac{\partial u}{\partial x}+\frac{\partial v}{\partial y}\right),
\end{gathered}
$$


and

$$
D E F=\sqrt{\left(\frac{\partial u}{\partial x}-\frac{\partial v}{\partial y}\right)^{2}+\left(\frac{\partial v}{\partial x}+\frac{\partial u}{\partial y}\right)^{2}} .
$$

Regions where the index was above a threshold were plotted and checked with pilot reports of CAT. The index was reasonably successful in estimating a few cases of CAT, though due to the sparsity of PIREPS, the regions of maximum TI could not be verified.

Keller (1986, 1990) proposed a Specific CAT Risk (SCATR) index based on frontogenesis. The Richardson number tendency equation is

$$
\frac{d \ln R i}{d d t}=-\Phi-\epsilon,
$$

where $\Phi$ is the resolvable or grid-scale component, and $\epsilon$ is the subgrid or 'turbulent' component. Using synoptic scale observations $\Phi$ was computed, while $\epsilon$ was parametrized in terms of velocity and temperature gradients. Using observed data for three documented cases of CAT, Keller showed that regions of positive $\Phi$ could explain the occurrence to a significant degree. The discrepancies were attributed to uncertainties in $\epsilon$.

Frehlich \& Sharman (2004) used a structure function approach, based on the observed spectra, to estimate small-scale turbulence using atmospheric variables from mesoscale NWP (numerical weather prediction) models.

Since none of the above indices have proven to be completely satisfactory, Sharman et al (2006), proposed the use of a combination of many indices of CAT with weights determined by recent PIREPS to calculate a 'Graphical Turbulence Guidance' system (GTG1). They use a numerical weather prediction model output at grid points (resolution of the order of 20-50 km) to calculate the probability of CAT. For the upper levels (> 20000 feet) 10 different indices, and for the middle levels (10000 to 20000 feet) nine indices are used. These include: Richardson number and windspeed (Endlich 1964); Turbulence Index 1 (Ellrod \& Knapp 1992); Colson-Panofsky index (Colson \& Panofsky 1965); frontogenesis function (Bluestein 1993); unbalanced flow diagnostic (Knox 1997); horizontal temperature gradient and structure function based estimate (Frehlich \& Sharman 2004).

These forecasts are available for the US region at the website http://adds.aviationweather.gov/ turbulence. A typical forecast is shown in figure 8.

Knox et al (2008) pointed out that a limitation of aviation turbulence forecasts was the identifying of sources of gravity waves and this could be part of the reason for the forecasts not achieving the U.S. Federal Government goals for CAT forecast techniques (probability of detection for moderate or greater turbulence greater than 0.8 , with a probability of detection of null reports greater than 0.8 ). On performing a scale analysis of the terms in equation 7 , they estimate that the leading order term is $f h \mathbf{u} \cdot \nabla \zeta$ and the second order terms are $-2 h \partial J(u, v) / \partial t, 2 h D f \zeta$ and $f h(v \partial D / \partial x-u \partial D / \partial y)$. Here $D$ is the horizontal divergence, $\zeta$ the relative vorticity, $u, v$ the horizontal velocity components and $J(u, v)=(\partial u / \partial x)(\partial v / \partial y)-(\partial v / \partial x)(\partial u / \partial y)$. Knox et al argue that the leading term, advection of relative vorticity, is consistent with other empirical approaches for predicting CAT. They have used an index based on the RHS terms and shown for some cases that it predicts a more localized region than other methods. Using similar arguments, the TI index of Ellrod \& Knapp (1992) has been modified to include a proxy term for divergence tendency and has been shown to improve CAT diagnostics (Ellrod \& Knox 2010).

To the objections raised by Plougonven et al (2009) regarding the scale separation in the application of the theory, Knox et al (2009) justify their approach citing the messy nature of real-life CAT forecasting. 


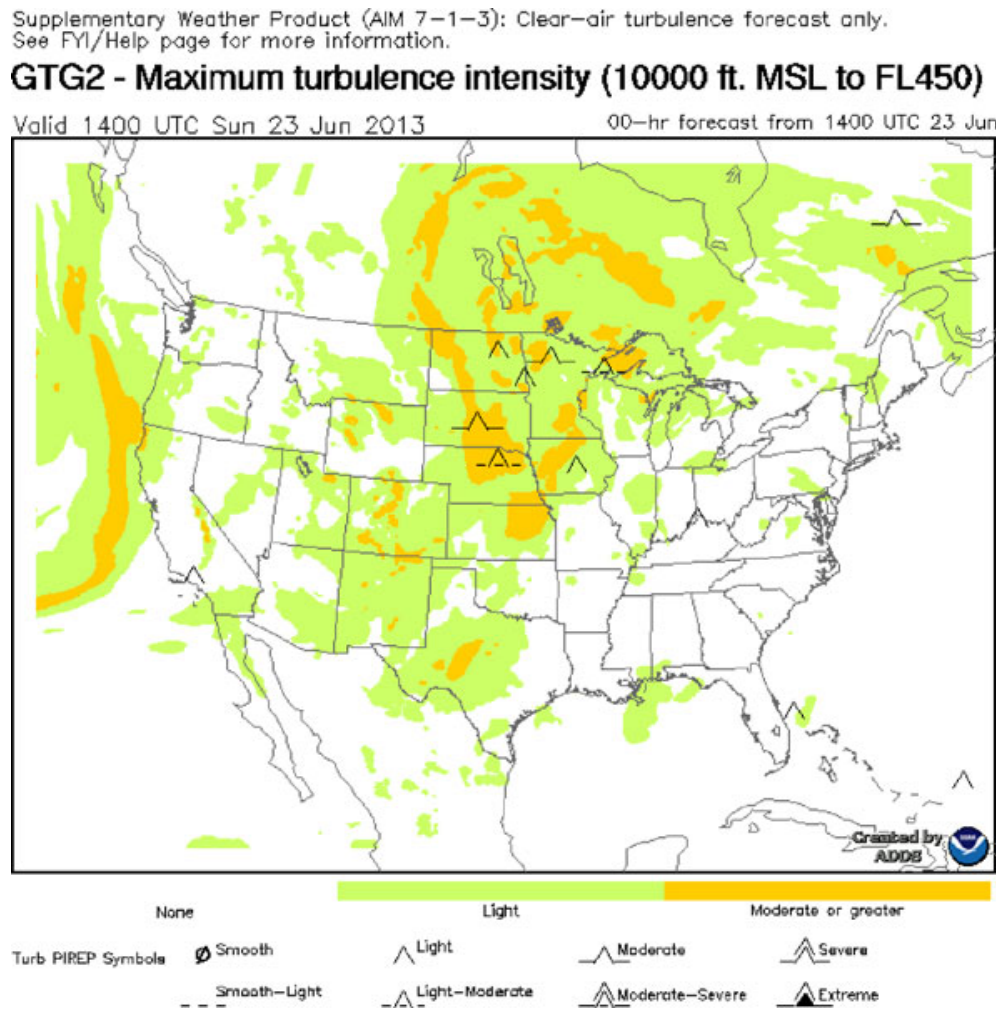

Figure 8. A sample of graphical turbulence guidance (GTG). URL: http://www.aviationweather.gov/ adds/data/turbulence/00_gtg_max.gif.

\section{Summary}

The problem of clear air turbulence has been widely studied since it was encountered by aircraft flying at altitudes above $5 \mathrm{~km}$. Being a phenomenon which occurs away from convective activity, its detection and measurement have been difficult. Observational studies have shown that CAT is associated with mesoscale-phenomena such as jet-streams, troughs, ridges, fronts. The flow conditions such as high vertical velocities, wind-shear, low Richardson number have been correlated with regions of CAT.

On the theoretical front, in the 1960s and 70s, the Kelvin-Helmholtz instability in a stably stratified fluid was considered to explain the formation of CAT (Atlas et al 1970). The Richardson number was therefore used as an index of CAT. Later, the importance of internal inertia-gravity waves was emphasized (Lindzen 1974; Ellrod et al 2003) and the role of mountains in triggering gravity waves was considered (Pao \& Goldburg 1969).

In the following decades, focus was on operational forecasting methods with a number of indices using combinations of Richardson number, vertical wind shear, horizontal convergence, deformation, etc. In the recent years, there has been a revival of interest in the theoretical aspects (Ford et al 2000; Knox et al 2008; Medvedev \& Gavrilov 1995). New mechanisms such as HVTs (Clark et al 2000) have also been suggested as sources of intense CAT. Among the theories based on inertia-gravity waves, the connection with actual location where CAT is encountered is often 
unclear. Among the 'local' theories ( $\mathrm{K}-\mathrm{H}$ instability, frontogenesis and spontaneous imbalance) the difficulty of detecting the trigger or critical perturbation remains.

While a variety of forecasting methods are being used, basic questions remain. The presence of a large number of different indices (around 10) in the GTG procedure of Sharman et al (2006), point out that there is no satisfactory theory. A difficulty in validation of different theories is the sparsity of PIREPs which remain the only sources of observation so far. In the future, satellite based data could offer global coverage which would make verification of theories easier.

As can be seen from the previous sections, there are a large number of mechanisms proposed for CAT. Given the different ways by which CAT is formed, it is likely that in each class there is a different mechanism in play. In our view, if we look at two ends of the spectrum, the possible mechanisms are: (i) mild turbulence-inertia-gravity waves and, (ii) severe turbulence-horizontal vortex tubes. Instead of trying to find a universal mechanism and prediction method for all forms of CAT, it may be fruitful to concentrate on the severe form, which is potentially more damaging, even though less frequent.

There is an evidence to suggest that generation and sustenance of horizontal vortex tubes of the appropriate scale, could be the source of severe clear air turbulence. For basic shear flows, a large number of studies have been conducted. It would be fruitful to explore the role of secondary structures in shear flows and connections of these to atmospheric flows.

Studies of the manner of sustenance/decay of CAT could be a promising area of research. A more holistic approach of the problem with the modelling of weather systems, land-sea processes, air traffic corridors and urban effects could be another area of research.

\section{References}

Aiken W S and Lean D (eds) 1973 Flight in Turbulence, AGARD Conference Proceedings No. 140

Atlas D, Metcalf J I, Richter J H and Gossard E E 1970 Birth of 'CAT' and Microscale Turbulence. J. Atmospher. Sci. 27: 903-913

Belcher S E and Hunt J C R 1998 Turbulent flow over hills and waves. Annual Review of Fluid Mechanics 30: $507-538$

Bluestein H B 1993 Observations and Theory of Weather Systems. Vol. II. Synoptic - Dynamic Meteorology in Midlatitudes. Oxford University Press, Oxford, UK, 594p

Chandrasekhar S 1961 Hydrodynamic and Hydromagnetic stability. Oxford University Press, Oxford, UK

Clark T L, Hall W D, Kerr R M, Middleton D, Radke L F, Ralph F M, Neiman P J and Levinson D 2000 Origins of Aricraft-Damaging Clear-Air Turbulence during the 9 December 1992 Colorado Downslope Windstorm: Numerical Simulations and Comparison with Observations. Journal of the Atmospheric Sciences 57: 1105-1131

Clark T L and Radke L F 2001 Clear Air Turbulence. ICAO journal 56(7): Page 5

Colson D 1963 Analysis of Clear Air Turbulence Data for March 1962. Monthly Weather Review 91: 73-82

Colson D and Panofsky H A 1965 An index of clear-air turbulence. Quarterly Journal of the Royal Meteorological Society 91: 507-513

Dickey T D and Mellor G L 1980 Decaying turbulence in neutral and stratified fluids. Journal of Fluid Mechanics 99(1): 13-31

Donovan M, Williams E, Kessinger C, Blackburn G, Herzegh P H, Bankert R L, Miller S D and Mosher F R 2008 The identification and verification of hazardous convective cells over oceans using visible and infrared satellite observations. Journal of Applied Meteorology and Climatology 47: 164-184

Drazin P G 2002 Introduction to Hydrodynamic Stability. Cambridge University Press, Cambridge, UK

Ellrod G P and Knapp D I 1992 An objective Clear-Air Turbulence Forecasting Technique: Verification and operational use. Weather and Forecasting 7: 150-165 
Ellrod G P and Knox J A 2010 Improvements to an Operational Clear-Air Turbulence Diagnostic Index by Addition of a Divergence Trend Term. Weather and Forecasting 25: 789-798

Ellrod G P, Lester P F and Ehernberger L J 2003 Clear Air Turbulence, in Encyclopedia of Atmospheric Sciences Vol 1., Holton J. R., Curry J. A. and Pyle (eds), Academic Press, California, USA, 393-403

Endlich R M 1964 The mesoscale structure of some regions of clear-air turbulence. Journal of Applied Meteorology 3: 261-276

Ford R 1994 Gravity wave radiation from vortex trains in rotating shallow water. Journal of Fluid Mechanics 281: 81-118

Ford R, McIntyre M E and Norton W A 2000 Balance and the Slow Quasimanifold: Some Explicit Results. Journal of the Atmospheric Sciences 57: 1236-1254

Frehlich R and Sharman R 2004 Estimates of turbulence from numerical weather prediction model output with applications to turbulence diagnosis and data assimilation. Monthly Weather Review 132: 23082324

Gavrilov N M 1997 Parametrization of momentum and energy depositions from gravity waves generated by tropospheric hydrodynamic sources. Ann. Geophysicae 15: 1570-1580

Howard L N 1961 Note on a paper pf John W. Miles. Journal of Fluid Mechanics 10: 509-512

Kauffmann P 2002 The business case for turbulence sensing systems in the US air transport sector. Journal of Air Transport Management 8: 99-107

Keller J L April 1986 The physical and empirical basis for a Specific Clear-Air Turbulence Risk Index, NASA-CR-3971

Keller J L 1990 Clear Air Turbulence as a response to meso and synoptic scale dynamic processes. Monthly Weather Review 118: 2228-2242

Knox J A 1997 Possible mechanisms of Clear-Air Turbulence in Strongly Anticyclonic Flows. Monthly Weather Review 125: 1251-1259

Knox J A, McCann D W and Williams P D 2008 Application of the Lighthill-Ford Theory of Spontaneous Imbalance to Clear-Air Turbulence Forecasting. Journal of the Atmospheric Sciences 65: 3292-3304

Knox J A, McCann D W and Williams P D 2009 Reply to Comments of Plougoven etal. Journal of the Atmospheric Sciences 66: 2511-2516

Kronebach G W 1964 An automated procedure for forecasting clear-air turbulence. Journal of Applied Meteorology 3(2): 119-125

Lin Yuh-Lang 2007 Mesoscale Dynamics. Cambridge University Press, Cambridge, UK

Lindzen R S 1974 Stability of a Helmholtz Velocity Profile in a Continuously Stratified, Infinite Boussinesq Fluid - Applications to Clear Air Turbulence. Journal of the Atmospheric Sciences 31: 1507-1514

Mahapatra P R, Mazur V, Doviak R J and Zrni D S 1999 Aviation Weather Surveillance Systems. Copublished by Institution of Electrical Engineers (UK) and American Institute of Aeronautics and Astronautics

Mancuso R L and Endlich R M 1966 Clear Air Turbulence frequency as a function of wind shear and deformation. Mon. Wea. Rev. 94: 581-585

Medvedev A S and Gavrilov N M 1995 The nonlinear mechanism of gravity wave generation by meteorological motions in the atmosphere. Journal of Atmospheric and Terrestrial Physics 57(11): $1221-1231$

Miles J W 1961 On the stability of heterogeneous shear flows. J. Fluid Mech. 10: 496-508

Moisy F, Morize C, Rabaud M and Sommeria J 2011 Decay laws, anisotropy and cycloneanticyclone asymmetry in decaying rotating turbulence. J. Fluid Mech. 666: 5-35

Nappo C J 2002 An Introduction to Atmospheric Gravity Waves. Academic Press, California, USA

Pao Y H and Goldburg A (eds) 1969 Clear Air Turbulence and its detection. Plenum Press, New York, USA

Plougonven R, Snyder C and Zhang F 2009 Comments on 'Application of the Lighthill-Ford Theory of Spontaneous Imbalance to Clear-Air Turbulence Forecasting'. Journal of the Atmospheric Sciences 66: 2506-2510

Sharman R, Tebaldi C, Wiener G and Wolff J 2006 An Integrated Approach to Mid and Upper-Level Turbulence Forecasting. Weather and forecasting 21: 268-287 
Simpson J E 1997 Gravity currents in the environment and the laboratory. Cambridge University Press, Cambridge, UK

Spalart P R 1996 On the motion of laminar wing wakes in a stratified fluid. J. Fluid Mech. 327: 139-160

Spalart P R 1998 Airplane trailing vortices. Annual Review of Fluid Mechanics 30: 107-138

Smyth W D and Winters K B 2003 Turbulence and Mixing in Holmboe Waves. J. Physical Oceanography 33: 694-711

Thorpe S A 1968 A Method of Producing a Shear Flow in a Stratified Fluid. J. Fluid Mech. 32: 693-704

Thorpe S A 1971 Experiments on the Instability of Stratified Shear Flows: Miscible Fluids. J. Fluid Mech. 46: 299-320

Thorpe S A 1973 Turbulence in stably stratified fluids: a review of laboratory experiments. Boundary-Layer Meteorology 5: 95-119

Weinstock J 1984 Effect of gravity waves on turbulence decay in stratified fluids. J. Fluid Mech. 140: 11-26 